\title{
Role of Intracellular Sodium Concentration and Autonomic Nervous Systems in Blood Pressure Regulation
}

\author{
Koji Matsumoto, Tetsuya Oshima*, Ryoji Ozono**, Hideo Matsuura**, Takafumi Ishida* ${ }^{* *}$ Mari Ishida**, \\ Katsuhiko Ishibashi ${ }^{* *}$, Mitsuaki Watanabe**, and Goro Kajiyama ${ }^{* *}$
}

\begin{abstract}
The purpose of the present study is to clarify the role of intracellular sodium concentration and the autonomic nervous system in the maintenance of blood pressure, and to examine genetic effects on these systems in young normotensives. First, the effects of sodium chloride intake on blood pressure and erythrocyte sodium concentration were examined in young normotensive men. The change in dietary sodium chloride from $50 \mathrm{mmol} /$ day to $340 \mathrm{mmol} /$ day induced no significant change in blood pressure or erythrocyte sodium concentration regardless of a family history of hypertension. The relationship between intraerythrocytic sodium concentration and blood pressure under pharmacological autonomic blockade was examined in normotensive subjects with and without a family history of hypertension under free sodium intake. After total autonomic blockade with atropine, propranolol and phentolamine, mean blood pressure did not change in normotensive subjects without a family history of hypertension $(81.0 \pm 8.6$ to $82.8 \pm 7.9 \mathrm{mmHg})$ but increased in normotensive subjects with a family history of hypertension $(84.8 \pm 9.9$ to $91.6 \pm 8.3 \mathrm{mmHg}, p<0.01)$. The difference in mean blood pressure between the two groups was significant only after autonomic blockade $(\boldsymbol{p}<\mathbf{0 . 0 5})$. Intraerythrocytic sodium concentration was higher in normotensive subjects with a family history of hypertension than in normotensive subjects without a family history of hypertension, but the difference was not significant. In the combined groups, intraerythrocytic sodium concentration was significantly correlated with mean blood pressure after total autonomic blockade $(r=0.68, p=0.003)$ rather than with basal blood pressure. These results suggest that intrinsic blood pressure, excluding the effects of the autonomic nervous systems may be associated with intracellular sodium concentration and that intrinsic blood pressure increased via elevated intracellular sodium level may be compensated for by the autonomic nervous system in normotensive subjects with a hereditary predisposition to hypertension. (Hypertens Res 1994; 17: 17-22)
\end{abstract}

Key Words: essential hypertension, family history, autonomic nervous system, sodium transport system, intrinsic blood pressure

It has been suggested that elevation in intracellular sodium concentration ([Na]i) may contribute to the pathogenesis of essential hypertension (1). Despite some controversial views $(2,3)$, a number of reports have demonstrated higher [Na]i levels in human essential hypertension (4-7). Also, normotensive offspring of patients suffering from essential hypertension, a group predisposed to high blood pressure later in life, manifest a higher $[\mathrm{Na}] \mathrm{i}$ in their red and white blood cells $(7-10)$.

However, it is difficult to establish a direct and close correlation between blood pressure and $[\mathrm{Na}] \mathrm{i}$ (7). One possiblility is that differences in sodium intake among individuals could mask the correlation. Thus, we first examined the effects of dietary sodium chloride on blood pressure and erythrocyte $[\mathrm{Na}] \mathrm{i}$ in young normotensives. Another reason for difficulty in defining the relationship may be that blood pressure is regulated by not only [Na]i but also by many other factors. Among these factors, the autonomic nervous system has a considerable effect on blood pressure regulation, and causes large variations in blood pressure $(11,12)$. We therefore performed pharmacological autonomic blockade in order to evaluate the roles of the autonomic nervous system and $[\mathrm{Na}] \mathrm{i}$ in maintaining blood pressure. In addition, we examined the influence of heredity of hypertension on these parameters since genetic factors may contribute to the pathogenesis of essential hypertension (13).

\section{Subjects and Methods}

Normotensive subjects were recruited from the

From the Department of Internal Medicine, Saiseikai Hiroshima Hospital, *Department of Clinical Laboratory Medicine and ${ }^{*}$ First Department of Internal Medicine, Hiroshima University School of Medicine.

Address for Reprints: Tetsuya Oshima, M.D., Department of Clinical Laboratory Medicine, Hiroshima University School of Medicine 1-2-3 Kasumi, Minami-ku, Hiroshima 734, Japan.

Received October 30, 1992; accepted in revised form September 21, 1993. 
male physicians on the staff of our laboratory. They had a blood pressure below $140 / 90 \mathrm{mmHg}$ when screened. They were selected based on whether or not they had a family history of hypertension. A positive family history of hypertension was defined as a case in whom at least one parent was undergoing treatment for hypertension. Relatives were considered normotensive only if there was no history of treatment for hypertension detected and their normal blood pressure was confirmed by physical checkup recording. No subjects had taken any drugs for at least 4 weeks before the study. The study protocol was explained to the subjects and their informed consent was obtained.

\section{Study Protocol}

Study 1. The Effects of Dietary Sodium Chloride Intake on Blood Pressure and Erythrocyte [Na]i

Twelve young nomotensives were enrolled into the study. Six had a positive family history of hypertension ( $30 \pm 3$ years old) and the remaining 6 had a negative family history $(31 \pm 4$ years old). After a week of a regular sodium chloride diet $(170 \mathrm{mmol} /$ day), all subjects were placed on a low sodium chloride diet (50 mmol/day) for one week and then a high sodium chloride diet $(340 \mathrm{mmol} /$ day $)$ for one week. A 24-hour urine collection was performed every day for determination of sodium and potassium excretions. On the seventh morning of each diet, the fasting subjects maintained a supine position for $30 \mathrm{~min}$. Then, blood pressure and pulse rate were measured and a venous blood sample was obtained for the determination of erythrocyte $[\mathrm{Na}] \mathrm{i}$ and hematocrit. Blood pressure measurements were performed with a sphygmomanometer every minute for $10 \mathrm{~min}$, and the average of 10 consecutive blood pressure readings was taken as the value for each diet period. Mean blood pressure was calculated as the sum of the diastolic blood pressure and onethird of the pulse pressure.

Study 2. The Eeffect of Pharmacological Autonomic Blockade on Blood Pressure

Ten subjects with a family history and 10 subjects without a family history of hypertension were studied. All subjects were allowed to eat and drink ad libtum. The examination was performed between 0900 and 1100 hours, following an overnight fast. After a catheter was inserted into the antecubital vein, the subjects then rested in a supine position for $30 \mathrm{~min}$ in a quiet room in the presence of only the examining physician. Conversation was avoided. Blood pressure was measured with a mercury sphygmomanometer, and heart rate by electrocardiography at one minute intervals throughout the examination. After these measurements, venous blood was drawn for the determination of $[\mathrm{Na}] \mathrm{i}$ in erythrocytes. Thereafter, intravenous injection of atropine sulfate $(0.04 \mathrm{mg} / \mathrm{kg}$ body weight, Tanabe Co., Osaka, Japan) and propranolol hydrochloride (0.2 mg/ kg, Sumitomo Co., Osaka, Japan) were given in sequence, each agent being administered over $5 \mathrm{~min}$. Measurements of blood pressure and heart rate were repeated 5 min after the administration of each compound. This regimen effectively blocks physiologic autonomic nervous influence on the heart (14). Finally, an alpha-adrenergic blocking drug, phentolamine mesylate $(0.2 \mathrm{mg} / \mathrm{kg}$, Ciba Geigy Co., Takarazuka, Japan), was injected intravenously for $30 \mathrm{sec}$, and measurements of blood pressure and heart rate were repeated 5 min later. Minimum blood pressure after total autonomic blockade was defined as intrinsic blood pressure.

\section{Measurements}

$[\mathrm{Na}] \mathrm{i}$ in erythrocytes was determined as previously reported (15). Briefly, $3 \mathrm{ml}$ of freshly drawn venous blood was collected into a heparinized tube and centrifuged at $1,600 \mathrm{~g}$ for $10 \mathrm{~min}$ at room temperature. After the plasma and buffy coat were removed, isolated erythrocytes were washed twice with cold isotonic $\mathrm{MgCl}_{2}$ solution and injected into a non-heparinized polycarbonate microcapillary tube (Hematolon, Kayagaki Irikakogyo, Tokyo, Japan) in which the length for a volume of $30 \mu \mathrm{l}$ had been premeasured. Following centrifugation at $15,000 \mathrm{~g}$ for $5 \mathrm{~min}$ at room temperature, $30 \mu \mathrm{l}$ of packed erythrocytes was transferred into $3 \mathrm{ml}$ of a hypotonic hemolysing solution containing $0.5 \%$ $\mathrm{LiCl}$. The concentration of sodium was determined by flame photometry (Hitachi, 775-A, Tokyo, Japan) in a supernatant of hemolytic solution. The percentage of extracellular volume trapped within the erythrocyte pellet determined by ${ }^{131}$ I-labelled human serum albumin was $2.5 \%$ and a correction was made for this value in $[\mathrm{Na}] \mathrm{i}$ in erythrocytes. Each measurement was made in triplicate and expressed as $\mathrm{mmol} / \mathrm{l}$ cells. The intra-assay coefficient of variation and day-to-day intrasubject variation in [Na]i were $1.4 \%$ and $1.8 \%$, respectively.

\section{Statistical Analysis}

All the data are shown as mean \pm SD. Statistical analyses were performed using Wilcoxon rank-sum test to compare the different study groups, and Wilcoxon matched-pairs signed rank test to compare between before and after total autonomic nervous blockade. Correlation coefficients were determined by Pearson correlations. $P$ value less than 0.05 was regarded as significant.

\section{Results}

Study 1. Effects of Dietary Sodium Chloride Diet on Blood Pressure and Erythrocyte [Na]i

Mean blood pressure, pulse rate, hematocrit and erythrocyte $[\mathrm{Na}] \mathrm{i}$ during each diet period are shown in Table 1. Mean blood pressure, pulse rate and hematocrit were similar in subjects with and without a family history. Erythrocyte $[\mathrm{Na}] \mathrm{i}$ was greater by about $9 \%$ in subjects with a positive family history than those with a negative family history for each diet; however, the difference was not significant $(0.05<p<0.10)$. No significant change was detected in any parameters, except urinary sodium excretion, with a change in sodium chloride intake in either group or the combined groups. No subject showed a change in mean blood pressure of more than 5 $\mathrm{mmHg}$ or a change in erythrocyte $[\mathrm{Na}] \mathrm{i}$ of more 
Table 1. Clinical Variables on Regular, Low and High Sodium Chloride Diets

\begin{tabular}{lcccc}
\hline Variable & FH & \multicolumn{3}{c}{ Sodium chloride diet } \\
\cline { 3 - 5 } & & Regular & Low & High \\
\hline Mean blood pressure (mmHg) & $(+)$ & $85.8 \pm 6.0$ & $84.8 \pm 4.8$ & $85.4 \pm 4.7$ \\
Pulse rate (beats/min) & $(-)$ & $83.6 \pm 8.0$ & $84.7 \pm 7.7$ & $84.5 \pm 8.0$ \\
& $(+)$ & $64 \pm 7$ & $62 \pm 6$ & $61 \pm 4$ \\
Hematocrit (\%) & $(-)$ & $64 \pm 6$ & $65 \pm 7$ & $63 \pm 7$ \\
Erythrocyte [Na]i & $(+)$ & $43.3 \pm 1.6$ & $43.4 \pm 1.1$ & $43.3 \pm 1.6$ \\
(mmol/l cells) & $(-)$ & $43.1 \pm 1.9$ & $43.2 \pm 2.8$ & $42.5 \pm 2.4$ \\
Urinary sodium (mmol/day) & $(+)$ & $8.77 \pm 0.80$ & $8.92 \pm 0.84$ & $8.88 \pm 0.76$ \\
& $(-)$ & $8.07 \pm 0.54$ & $8.13 \pm 0.56$ & $8.12 \pm 0.52$ \\
& $(-)$ & $166 \pm 10$ & $50 \pm 7$ & $329 \pm 20$ \\
\hline
\end{tabular}

$\mathrm{FH}$, family history of hypertension; $[\mathrm{Na}] \mathrm{i}$, intracellular sodium concentration.

than $0.2 \mathrm{mmol} / 1$ cells. In the total subjects, there was no significant correlation between erythrocyte $[\mathrm{Na}] \mathrm{i}$ and mean blood pressure for any diet $(r=$ $0.34,0.34$ and 0.43 in regular, low and high salt diets, respectively).

Study 2. Effect of Pharmacological Autonomic Blockade on Blood Pressure and Erythrocyte [Na]i Characteristics of subjects with and without a family history of hypertension are shown in Table 2. There was no difference in age or body weight. No significant difference was detected in basal blood pressure between the two groups. $[\mathrm{Na}] \mathrm{i}$ in erythrocytes was higher by $10 \%$ in the former group than in the latter, but this difference was not statistically significant $(p=0.09)$, similar to Study 1 .

Figure 1 shows the effect of pharmacological autonomic blockade on mean blood pressure. With total autonomic blockade using atropine, propranolol and phentolamine, mean blood pressure did not change in the group without a family history $(81.0 \pm$ 8.6 to $82.8 \pm 7.9 \mathrm{mmHg}$ ); in contrast, it increased in the group with a family history $(84.8 \pm 9.9$ to $91.6 \pm$ $8.3 \mathrm{mmHg}, p<0.01)$. A significant intergroup difference in blood pressure was detectable only after total autonomic blockade $(p<0.05)$. When the effect of each drug was evaluated separately, atropine increased and phentolamine decreased mean blood pressure in each group; propranolol did not affect mean blood pressure. The change in mean blood pressure by each blockade was not significantly different between the groups.

Figure 2 shows the relation of $[\mathrm{Na}] \mathrm{i}$ in erythrocytes to mean blood pressure before and after total autonomic blockade. Erythrocyte [Na]i was positively correlated with both blood pressure levels. Correlation between erythrocyte $[\mathrm{Na}] \mathrm{i}$ and mean blood pressure was closer after total autonomic blockade $(r=0.68, p=0.003)$ than that before $(r=$ $0.53, p=0.03)$.

\section{Discussion}

Intracellular sodium accumulation (4-7) and abnormalities in cellular sodium transport systems (4, 16-18) have been frequently reported in patients with essential hypertension. These factors have been proposed to be involved in the pathogenesis of this disease (1). We also have demonstrated that $[\mathrm{Na}] \mathrm{i}$ in erythrocytes (7) and lymphocytes $(6,15)$ were higher in patients with essential hypertension than in normotensive controls. Furthermore, our previous report showed that normotensive subjects with a positive family history of hypertension had a higher [Na]i in erythrocytes than subjects with a negative family history (7). Thus, intracellular sodium accumulation may precede the development of hypertension. However, as the overlap between [Na]i in erythrocytes of each group was great (7), a large population study would most likely be necessary to detect significant intergroup differences. We

Table 2. Characteristics of Subjects in Study 2

\begin{tabular}{lcc}
\hline & FH - & FH+ \\
Variable & $(n=10)$ & $(n=10)$ \\
\hline Age (year) & $30 \pm 5$ & $29 \pm 4$ \\
Body Weight (kg) & $64 \pm 8$ & $65 \pm 5$ \\
Mean Blood Pressure (mmHg) & $81.1 \pm 8.6$ & $84.8 \pm 9.9$ \\
Heart Rate (beats/min) & $60 \pm 11$ & $61 \pm 14$ \\
Erythrocyte [Na]i (mmol/l cells) & $7.97 \pm 0.69$ & $8.80 \pm 1.32$ \\
\hline
\end{tabular}

$\mathrm{FH}-$, normotensive subjects without a family history of hypertension; $\mathrm{FH}+$, normotensive subjects with a family history of hypertension; [Na]i, intracellular sodium concentration. 


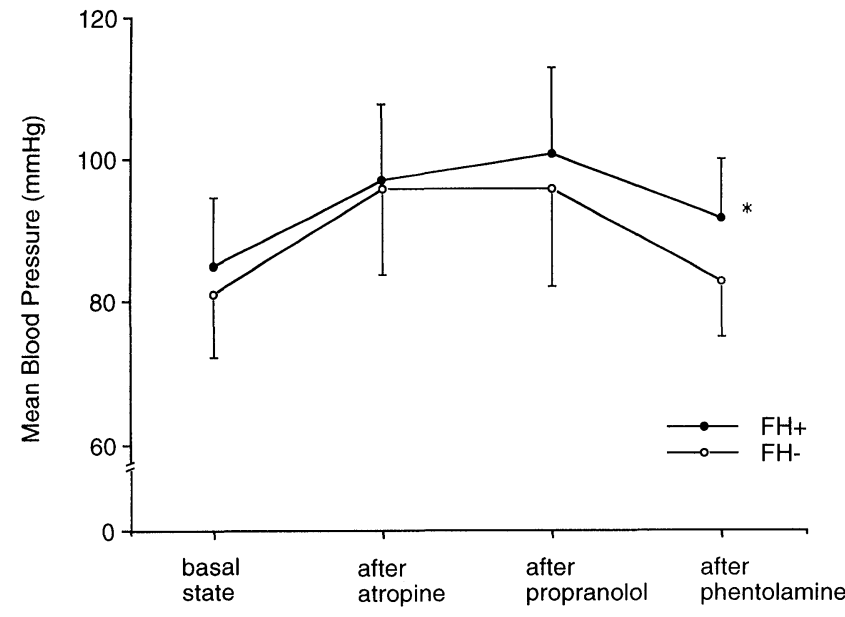

Fig. 1. Effect of pharmacological autonomic blockade on mean blood pressure in normotensive subjects with (0) and without (O) a family history of hypertension $(\mathrm{FH}+$ and $\left.\mathrm{FH}^{-}\right) .{ }^{*} \mathrm{p}<0.05$ between groups.

could not detect a statistically significant difference between the subjects with and without a family history in the present study (Study 1 and Study 2) where relatively few subjects were used, although $[\mathrm{Na}] \mathrm{i}$ in the group with a positive family history was higher by $9-10 \%$ than that in the group with a negative family history.

In the present study, a positive and close correlation could be detected between $[\mathrm{Na}] \mathrm{i}$ in erythrocytes and intrinsic mean blood pressure, regarded as the blood pressure after total autonomic nervous blockade. A weak link could be found between [Na]i and blood pressure without any manipulation in our previous study (7) and the present study. Thus, [Na]i may be one of the most important factors involved in blood pressure regulation when the effect of the autonomic nervous system on blood pressure is removed. Blood pressure is regulated by

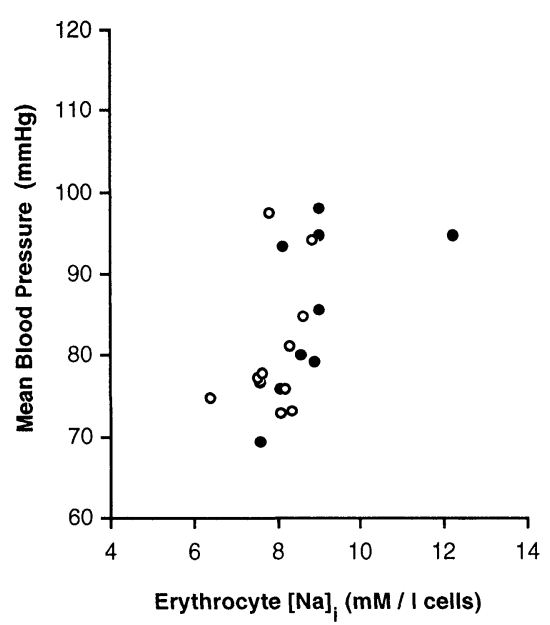

multiple factors such as the sympathetic and parasympathetic nervous systems, the renin-angiotensinaldosterone system, body fluid balance and cellular cation metabolism. The importance of the autonomic nervous system is supported by clinical evidence of the significant antihypertensive efficacy of adrenergic inhibiting drugs and by the observation that variations in blood pressure are largely dependent on sympathetic nerve activity $(11,12)$. Therefore, our present study findings suggest that the autonomic nervous system may obscure the close correlation between [Na]i and intrinsic blood pressure.

One other important finding is the genetic effect on the role of the autonomic nervous system in blood pressure regulation. In the present study, whereas total autonomic nervous blockade resulted in an elevation of blood pressure in subjects with a positive family history, it did not modify the blood pressure in subjects with a negative family history. Thus, the significant difference in blood pressure between groups could be detected only after the inhibition of total autonomic nervous activity. This finding may be able to explain why young offsprings of parents with hypertension have normal blood pressure although they share several abnormalities of essential hypertensive patients such as abnormal cellular sodium transport $(7,16-18)$, abnormal renal hemodynamics (19), suppressed renin-angiotensinaldosterone system (19) and an enhanced pressor response to mental stress $(20)$. The present results suggest that an increased intrinsic blood pressure in normotensive subjects with a hereditary predisposition to hypertension may be compensated by the autonomic nervous systems. However, it is difficult to decide which of the autonomic nervous systems contributes to the intergroup difference, because the effect of each blockade was not significantly different between the groups. However, the change in blood pressure by $\alpha$ - and $\beta$-blockade tended to be larger, whereas that by atropine tended to be smaller in subjects with a positive family history. It is

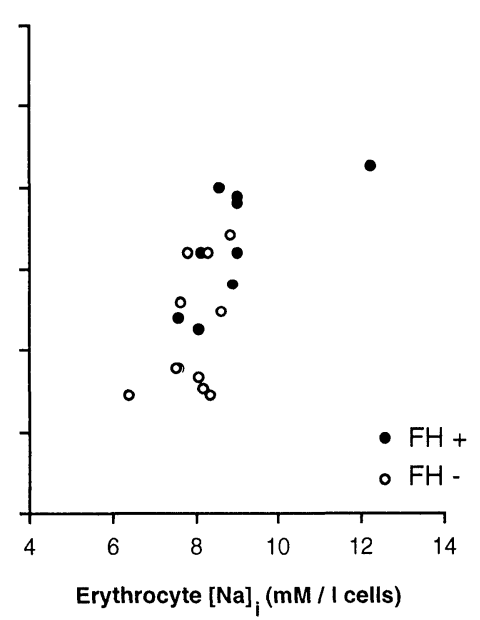

Fig. 2. Relationship between mean blood pressure before (left) and after (right) pharmacological autonomic blockade and intracellular sodium concentration ([Na]i) in erythrocytes. 
thus possible that the sympathetic rather than the parasympathetic nervous system may be responsible for the intergroup difference in mean blood pressure.

The precise mechanism whereby intracellular sodium accumulation raises intrinsic blood pressure is not fully clarified. One possible explanation is that an increased $[\mathrm{Na}] \mathrm{i}$ may raise $\left[\mathrm{Ca}^{2+}\right] \mathrm{i}$ through the $\mathrm{Na}^{+}-\mathrm{Ca}^{2+}$ exchange process and result in vasoconstriction, as proposed by Blaustein (21). Our previous studies showing that a close and positive correlation was observed between $[\mathrm{Na}] \mathrm{i}$ and $\left[\mathrm{Ca}^{2+}\right] \mathrm{i}$ in lymphocytes of humans (6), and that an increase in $[\mathrm{Na}] \mathrm{i}$ with dietary sodium chloride loading was accompanied by an increase in $\left[\mathrm{Ca}^{2+}\right] \mathrm{i}$ and an elevation in blood pressure in patients with essential hypertension (22) may support this possibility.

In study 1 , we confirmed that the change in sodium chloride intake did not induce a significant change in blood pressure or erythrocyte $[\mathrm{Na}] \mathrm{i}$ in young normotensive subjects. This result is consistent with the previous reports (23-25), although other investingators reported that $[\mathrm{Na}] \mathrm{i}$ is reduced (9) or increased (26) by sodium repletion. The different results among researchers may be due to differences in the amount of sodium chloride intake, the duration of dietary manipulation, and genetic factors. Interestingly, using the same methods of sodium chloride manipulation, we have previously shown the ability of sodium chloride loading to induce blood pressure elevation, intracellular sodium accumulation and volume expansion in patients with essential hypertension $(27,28)$. Subjects in the present study were young normotensives, whereas subjects in our previous studies $(23,24)$ were essential hypertensive patients with a mean age of about 50 years old. One may consider that the different effects of dietary salt result from different age and/ or blood pressure between our previous investigations and the present study. Because the effects of sodium chloride loading on blood pressure, erythrocyte $[\mathrm{Na}] \mathrm{i}$ and circulating plasma volume estimated by hematocrit are minimal in young normotensives, it is unlikely that the different sodium chloride intake affected the result in study 2.

In conclusion, we found that $[\mathrm{Na}] \mathrm{i}$ in erythrocytes had a close and positive correlation with mean blood pressure after total autonomic nervous blockade, and that this pharmacological manipulation resulted in blood pressure elevation in subjects with a family history of hypertension but not in those without a family history. These results suggest that $[\mathrm{Na}] \mathrm{i}$ may play a role in the determination of intrinsic blood pressure and that an increased intrinsic blood pressure via an increased [Na]i in normotensives with a hereditary predisposition to hypertension may be compensated by the autonomic nervous system.

\section{Acknowledgement}

The authors thank Miss Yuko Omura for her help in the preparation of this manuscript.

\section{References}

1. Swales JD: Ion transport in hypertension. Biosci Rep 1982; 2: 967-990.

2. Parker JC, Berkowitz LR: Physiologically instructive genetic variants involving the human red cell membrane. Physiol Rev 1983; 63: 261-313.

3. Simon G, Conklin DJ: In vivo erythrocytes sodium concentration in human hypertension is reduced, not increased. J Hypertens $1986 ; 4$ : 71-75.

4. Clegg G, Morgan DB, Davidson C: The heterogeneity of essential hypertension-Relation between lithium efflux and sodium content of erythrocytes and a family history of hypertension. Lancet 1976; ii: 891-894.

5. Tedde R, Sechi LA, Marigliano A, Campus S: Sodium Concentration in eythrocytes in arterial hypertension. J Hypertens 1983; 1: 140-141.

6. Oshima $\mathrm{T}$, Matsuura $\mathrm{H}$, Matsumoto $\mathrm{K}$, et al: Intralymphocytic sodium and free calcium and plasma renin in essential hypertension. Hypertension 1988; 12: $26-31$

7. Matsumoto $\mathrm{K}$, Matsuura $\mathrm{H}$, Oshima $\mathrm{T}$, et al: The significance of erythrocyte sodium contents on blood pressure: evaluation by multivariate analysis $[\mathrm{Ab}-$ stract]. Circulation 1986; 74-II: 488.

8. Nelson D, Henningson NC: Erythrocyte contents of electrolytes ( $\mathrm{Na}, \mathrm{K}, \mathrm{Mg}, \mathrm{Zn}$ ) in healthy male controls and offspring to established hypertensive patients: a follow-up study. Scand J Clin Lab Invest 1983; 43: 317-322.

9. Gudmondson $\mathrm{O}$, Andersson $\mathrm{O}$, Herlitz $\mathrm{H}$, et al: Blood pressure, intraerythrocyte content, and transmembrane fluxes of sodium during normal and high salt intake in subjects with and without a family history of hypertension: evidence against a sodium transport inhibitor. J Cardiovasc Pharmacol 1984; 6 (Suppl 1): s35-s41.

10. Lijnen $\mathrm{P}, \mathrm{M}$ 'Buyamba-Kabangu JR, Fagard RH, et al: Intracellular concentration and transmembrane fluxes of sodium and potassium in erythrocytes of white normal male subjects with and without a family history of hypertension. J Hypertens 1984; 2: 25-30.

11. Conway J, Boon N, Davies C, et al: Neural and hormonal mechanisms in blood pressure variavility. $J$ Hypertens 1984; 2: 203-208.

12. Eisenhofer G, Lambie DG, Johnson RH: $\beta$-adrenoceptor responsiveness and plasma catecholamines as determinants of cardiovascular reactivity to mental stress. Clin Sci 1985; 69: 483-492.

13. Watt G: Design and interpretation of studies comparing individuals with and without a family history of high blood pressure. J Hypertens 1986; 4: 1-7.

14. Jose AD, Taylor RR: Autonomic blockade by propranolol and atropine to study intrinsic myocardial function in man. J Clin Invest 1969; 48: 2019-2031.

15. Oshima $\mathrm{T}$, Matsuura $\mathrm{H}$, Matsumoto $\mathrm{K}$, et al: Intracellular sodium concentration in lymphocytes and erythrocytes of paients with essential hypertension. Jpn Heart J 1988; 29: 271-278.

16. Heagerty AM, Milner M, Bing RF, et al: Leukocyte membrane sodium transport in normotensive populations: dissociation of abnormalities of sodium efflux from raised blood-pressure. Lancet 1982; ii: 894-896.

17. Garay RP, Dagher G, Pernollet MG, et al: Inherited defect in $\mathrm{a} \mathrm{Na}^{+}, \mathrm{K}^{+}$, co-transport system in erythrocytes from essenial hypertensive patients. Nature 1980; 284: 281-283.

18. Canessa M, Adragna N, Solomon HS, et al: Increased sodium lithium countertransport in red cells of patients with essential hypertension. $N$ Engl J Med 1980; 302: 772-776.

19. van Hooft IMS, Grobbee DE, Derky FHM, et al: 
Renal hemodynamics and the renin-angiotensinaldosterone system in normotensive subjects with hypertensive and normotensive parents. $N$ Engl $J$ Med 1991; 324: 1305-1311.

20. Falkner B, Onesti G, Angelakos ET, et al: Cardiovascular response to mental stress in normal adolescents with hypertensive parents. Hypertension 1979; 1: 23-30.

21. Blaustein MP: Sodium ions, Calcium ions, blood pressure regulation, and hypertension: a reassessment and a hypothesis. Am $J$ Physiol 1977; 232: C165-C173.

22. Oshima $\mathrm{T}$, Matsuura $\mathbf{H}$, Matsumoto $\mathrm{K}$, et al: Intralymphocytic sodium and free calcium concentration in relation to salt sensitivity in patients with essential hypertension. Jpn Circ J 1987; 51: 1184-1191.

23. Cooper R, Trevisan M, Horn LV, et al: Effect of dietary sodium reduction on red blood cell sodium concentration and sodium-lithium countertransport. Hypertension 1984; 6: 731-735.

24. Fitzgibbon WR, Morgan TO, Myers JB: Salt sensitiv- ity of normotensives: interaction between changes in red blood cell ${ }^{22} \mathrm{Na}$ efflux rate constant, dietary sodium intake and changes in blood pressure. Clin Exp Pharmacol Physiol 1982; 9: 291-295.

25. Quintanilla AP, Weffer MI, Koh H, et al: Effect of high salt intake on sodiu, potassium-dependent adenosine triphosphatase activity in the erythrocytes of normotensive men. Clin Sci 1988; 75: 167-170.

26. Lijnen P, M'Buyamba-Kabangu JR, Fiocchi R, et al: Sodium and potassium fluzes and concentrations in erythrocytes of normal subjects during prolonged sodium depletion and repletion. Prostgrad Med $J$ 1986; 62: 3-12.

27. Oshima T, Matsuura H, Matsumoto K, et al: Factors determining sodium chloride sensitivity of patients with essential hypertension: Evaluation by multivariate analysis. J Hypertens 1989; 7: 223-227.

28. Oshima $\mathrm{T}$, Matsuura $\mathrm{H}$, Ishibashi $\mathrm{K}$, et al: Familial influence upon $\mathrm{NaCl}$ sensitivity in patients with essential hypertension. $J$ Hypertens 1992; 10: 1089-1094. 